Research article

\title{
Germline polymorphisms in SIPA1 are associated with metastasis and other indicators of poor prognosis in breast cancer
}

\author{
Nigel PS Crawford ${ }^{1 *}$, Argyrios Ziogas ${ }^{2 *}$, David J Peel ${ }^{2}$, James Hess ${ }^{2}$, Hoda Anton-Culver ${ }^{2}$ and \\ Kent W Hunter ${ }^{1}$
}

\author{
${ }^{1}$ Laboratory of Population Genetics, National Cancer Institute/National Institutes of Health, Bethesda, Maryland, USA \\ ${ }^{2}$ Epidemiology Division, Department of Medicine, University of California, Irvine, California, USA \\ * Contributed equally
}

Corresponding author: Kent W Hunter, hunterk@mail.nih.gov

Received: 4 Nov 2005 Revisions requested: 17 Jan 2006 Revisions received: 15 Feb 2006 Accepted: 20 Feb 2006 Published: 21 Mar 2006

Breast Cancer Research 2006, 8:R16 (doi:10.1186/bcr1389)

This article is online at: http://breast-cancer-research.com/content/8/2/R16

(c) 2006 Crawford et al.; licensee BioMed Central Ltd.

This is an open access article distributed under the terms of the Creative Commons Attribution License (http://creativecommons.org/licenses/by/2.0), which permits unrestricted use, distribution, and reproduction in any medium, provided the original work is properly cited.

\begin{abstract}
Introduction There is growing evidence that heritable genetic variation modulates metastatic efficiency. Our previous work using a mouse mammary tumor model has shown that metastatic efficiency is modulated by the GTPase-activating protein encoded by Sipa1 ('signal-induced proliferationassociated gene $\left.1^{\prime}\right)$. The aim of this study was to determine whether single nucleotide polymorphisms (SNPs) within the human SIPA1 gene are associated with metastasis and other disease characteristics in breast cancer.

Method The study population $(n=300)$ consisted of randomly selected non-Hispanic Caucasian breast cancer patients identified from a larger population-based series. Genomic DNA was extracted from peripheral leukocytes. Three previously described SNPs within SIPA1 (one within the promoter [-313G $>A$ ] and two exonic [545C $>T$ and $2760 G>A]$ ) were characterized using SNP-specific PCR.
\end{abstract}

Results The variant $2760 \mathrm{G}>\mathrm{A}$ and the $-313 \mathrm{G}>\mathrm{A}$ allele were associated with lymph node involvement $(P=0.0062$ and $P=$ 0.0083 , respectively), and the variant $545 \mathrm{C}>\mathrm{T}$ was associated with estrogen receptor negative tumors $(P=0.0012)$ and with progesterone negative tumors $(P=0.0339)$. Associations were identified between haplotypes defined by the three SNPs and disease progression. Haplotype 3 defined by variants $-313 \mathrm{G}>\mathrm{A}$ and $2760 \mathrm{G}>\mathrm{A}$ was associated with positive lymph node involvement $(P=0.0051)$, and haplotype 4 defined by variant $545 \mathrm{C}>\mathrm{T}$ was associated with estrogen receptor and progesterone receptor negative status $(P=0.0053$ and $P=$ 0.0199 , respectively).

Conclusion Our findings imply that SIPA1 germline polymorphisms are associated with aggressive disease behavior in the cohort examined. If these results hold true in other populations, then knowledge of SIPA1 SNP genotypes could potentially enhance current staging protocols.

\section{Introduction}

Breast cancer is a major public health concern among Western female populations. In 2004 breast cancer was the most common form of malignancy diagnosed in females in the USA and the second leading cause of cancer mortality in women [1]. The great majority of these deaths are related to complications caused by metastatic disease, and in spite of therapeutic advances metastatic breast cancer is currently incurable [2]. It has been estimated that $24-30 \%$ of women with node-negative disease and at least $50-60 \%$ of those with node-positive disease at diagnosis will relapse [3]. Furthermore, approximately $6-10 \%$ of breast cancer patients present with clinical evidence of metastasis, and the median survival of those with metastatic disease is estimated to be 2-4 years [3].

The impact of metastatic disease on prognosis underscores the importance of improving upon currently available means of diagnosing and treating advanced breast cancer. One potentially powerful approach would be to identify early those patients who are at high risk for disseminated disease and then administer tailored treatment to reduce or eliminate the emergence of secondary lesions. As our knowledge of both the genetic and molecular mechanisms underpinning metastasis improves, it is becoming apparent that use of simple, relia-

$\mathrm{Cl}=$ confidence interval; $\mathrm{D}^{\prime}=$ Lewontin's $\mathrm{D}$ prime; $\mathrm{ER}=$ estrogen receptor; $\mathrm{NCBI}=$ National Center for Biotechnology Information; OR = odds ratio; $\mathrm{PCR}=$ polymerase chain reaction; $\mathrm{PR}=$ progesterone receptor; $\mathrm{SNP}=$ single nucleotide polymorphism. 
ble and robust assays that rely upon stable, constitutional fingerprints, including germline DNA polymorphisms, may provide an alternative means of identifying patients at risk for advanced disease. Recent studies have demonstrated that low penetrance germline polymorphisms influence overall susceptibility to breast cancer (for review, see the report by Houlston and Peto [4]) as well as the likelihood of tumor progression and/or recurrence (for review, see the report by Foulkes and coworkers [5]). Probably the best documented genes with respect to the latter are BRCA1 and BRCA2, with specific germline mutations being demonstrated to be associated with various indicators of poor outcome [6-9].

Previously, our laboratory provided evidence that the host genetic background upon which a tumor arises can significantly alter metastatic efficiency. This was demonstrated by a breeding scheme in which the highly metastatic polyoma middle $\mathrm{T}$ transgenic mouse mammary tumor model was bred to a variety of inbred strains and the metastatic capacity of the tumors in each of the $F_{1}$ hybrid populations determined [10]. Wide variations in metastatic efficiency for each of the different $F_{1}$ progeny were observed following mammary tumor development [10], and because all of the tumors were induced by the same transgenic event - namely the activation of the polyoma middle $\mathrm{T}$ antigen [11] - it was concluded that the observed difference in metastatic efficiency in $\mathrm{F}_{1}$ progeny was a consequence of germline genetic variation between each of the different inbred strains.

Quantitative trait mapping experiments revealed the presence of a metastasis efficiency modifier locus linked to the proximal end of mouse chromosome 19 [12]. Subsequent evaluation of this locus identified a gene called Sipa1 ('signal-induced proliferation-associated gene 1'; also known as Spa1) as a likely candidate for this modifier locus. Sequence analysis of Sipa1 revealed an alanine to threonine mutation in the PDZ domain in mice with high metastatic potential (FVB strain, AKR strain) when compared with low metastatic phenotype mice (NZB strain, DBA strain) [13]. Computer modeling of the wild-type and mutant forms of Sipa1 indicated that this mutation occurs on the open face of an $\alpha$-helix, and in vitro analysis demonstrated that the polymorphism functionally impacts on proteinprotein interactions. Furthermore, these data indicated that the metastatic capacity of mammary tumors in spontaneous metastasis assays was significantly altered by modulation of Sipa1 expression. Metastatic capacity was increased by Sipa1 upregulation as a consequence of ectopic gene expression [13], and a decrease in metastasis was seen when Sipa1 expression was reduced by RNA interference utilizing Sipa1specific short-hairpin RNAs.

The aim of this study is to determine whether SIPA1 (MIM\# 602180 ) plays a similar role in the modulation of metastatic potential in human breast cancer populations. We hypothesized that polymorphisms or haplotypes within key regulatory or coding elements of the human gene encoding SIPA1, located on chromosome 11q13.3, are positively correlated with the presence of metastases in breast cancer patients. To test this hypothesis, we designed a case-only association study in which a number of single nucleotide polymorphisms (SNPs) in SIPA1 were characterized in patients with metastatic or nonmetastatic breast cancer.

\section{Materials and methods Recruitment of patients}

This study was approved by the institutional review boards of the US National Institutes of Health and the University of California (Irvine, CA, USA). Written informed consent was obtained from all patients. The patients included in this study are a subgroup of 300 women with breast cancer selected from a larger population-based population of incident breast cancer patients. Breast cancer probands diagnosed between 1 March 1994 and 28 February 1995 were identified through the population-based cancer registry of the Cancer Surveillance Program of Orange County. Description of the Program and details of data collection methods were reported previously [14]. The overall goal of the parent study was to provide a means to identify a large fraction of the population that is at genetically high risk for breast or ovarian cancer, and to determine the significance of inherited cancer-predisposing genes as risk factors for cancer development in the population.

We report here data on 300 breast cancer patients (all nonHispanic white women) selected from among the patients included in the parent study as follows: approximately $51 \%$ of patients with regional or metastatic breast cancer (154 cases); and randomly selected patients with localized disease (146 cases). Regional disease indicates direct extension of the primary tumor to the skin, muscle, chest wall, lymph nodes, or a combination of the above; metastatic disease indicates all other forms of involvement beyond regional disease. Pathologic diagnoses were obtained at the time of initial presentation in all cases, and the methods used for data collection were standardized. The protocol included initial contact with patients' physician followed by a mail-based invitation and description of the study. After patients had consented to participate in the study, family history and epidemiologic risk factors were assessed by telephone interview. In addition, data on tumor characteristics and stage at diagnosis were collected from the cancer registry. These data were based on pathology reports and medical records of the cancer patients. Blood samples were collected from all cancer patients, DNA was extracted from each sample, and aliquots were sent to the $\mathrm{NCl}$ (IRB \#HS-2004-3832).

\section{Selection of single nucleotide polymorphisms}

We characterized three SNPs within the regulatory or coding regions of SIPA1. SNPS were chosen on the basis of their genomic location from the NCBI SNP database [15]. One polymorphism, $-313 G>A$ (NCBI SNP designation rs931127) is 
Table 1

\begin{tabular}{|c|c|c|}
\hline SIPA1 polymorphism & & Sequence ${ }^{a}$ \\
\hline \multirow[t]{4}{*}{$-313 G>A(r s 931127)$} & Forward primer & 5'-GCGCGCTAGTGGAATGGTT-3' \\
\hline & Reverse primer & 5'-GACCAACCGGGAGGGCTA-3' \\
\hline & Wild-type probe & VIC-AGGGCCCAGTGCT \\
\hline & Variant probe & FAM-AGGGCCCAGTACTTA \\
\hline \multirow[t]{4}{*}{$545 \mathrm{C}>\mathrm{T}$ (F182S; rs3741378) } & Reverse primer & 5'-CCAGCTCGGACCTGCTGCAT-3' \\
\hline & Reverse primer & 5'-GATGGACACGGCCGCGTT-3' \\
\hline & Wild-type probe & VIC-ACCAGCATCCCCACC \\
\hline & Variant probe & FAM-ACCAGCATTCCCAC \\
\hline \multirow[t]{4}{*}{$2760 G>A(A 920 A ;$ rs746429) } & Forward primer & 5'-CCTTGTGCCCACAGTCATGTC-3' \\
\hline & Reverse primer & 5'-CGGGACAGCGACTCCAGAATG-3' \\
\hline & Wild-type probe & VIC-CACTGCCCGCCTC \\
\hline & Variant probe & FAM-CCСАCTGССТGCC \\
\hline
\end{tabular}

aFAM, 5-(\&6)-carboxyfluorescein; SNP, single nucleotide polymorphism; VIC, Applied Biosystems ${ }^{\circledR}$ proprietary dye.

located 313 base pairs upstream of the 5 '-untranslated region of SIPA1 and is considered to be within the promoter region of the gene. The remaining two SNPs are located within coding regions, one within exon 1 (545C>T [F182S]; rs3741378) and one within exon 12 (2760G>A [A920A]; rs746429).

\section{Single nucleotide polymorphism genotyping}

SIPA1 polymorphisms were characterized using SNP-specific PCR. The PCR primers were designed using Vector NTI 9.0 software (Invitrogen, Carlsbad, CA, USA), in accordance with parameters described elsewhere [16]. Each probe was labeled with a reporter dye (either $\mathrm{VIC}^{\circledR}$ [a proprietary fluorescent dye produced by Applied Biosystems, Foster City, CA, USA] or FAM [5-(\&6)-carboxyfluorescein]) specific for wildtype and variant alleles of each of three SIPA1 SNPs, respectively. Sequences of PCR primers and fluorogenic probes are given in Table 1.

Reaction mixtures consisted of $300 \mathrm{nmol} / \mathrm{l}$ of each oligonucleotide primer, $100 \mathrm{nmol} / \mathrm{l}$ fluorogenic probes, $8 \mathrm{ng}$ template DNA, and 2× TaqMan Universal PCR Master Mix (Applied Biosystems) in a total volume of $10 \mu \mathrm{l}$. The amplification reactions were performed in a MJ Research DNA Engine thermocycler (Bio-Rad, Hercules, CA, USA) with two initial hold steps $\left(50^{\circ} \mathrm{C}\right.$ for 2 minutes, followed by $95^{\circ} \mathrm{C}$ for 10 minutes) and 40 cycles of a two-step PCR $\left(92^{\circ} \mathrm{C}\right.$ for $15 \mathrm{~s}, 60^{\circ} \mathrm{C}$ for 1 minute). The fluorescence intensity of each sample was measured post-PCR in an ABI Prism 7700 sequence detection system (Applied Biosystems), and SIPA1 SNP genotypes were determined by the fluorescence ratio of the nucleotide-specific fluorogenic probes.

\section{Statistical analysis}

We used Student's $t$ test to compare means for continuous variables and Wilcoxon's sum rank test to compare medians. Variables that were not normally distributed such as tumor size were log transformed. $\chi^{2}$ test or Fisher's exact tests were used to test for differences between categorical variables and testing for Hardy-Weinberg equilibrium. Unconditional logistic regression adjusting for multivariate covariates such as age at diagnosis was used to estimate the adjusted odds ratios (ORs). Lewontin's D prime ( $\left.D^{\prime}\right)$ and correlation coefficient $\left(r^{2}\right)$ were calculated as two measures of linkage disequlibrium. Using the E-M algorithm [17], we estimated frequencies of the most common haplotypes (with a frequency $>1 \%$ ) in this population and we imputed expected haplotypes for each subject. Haplotype specific ORs were calculated from unconditional logistic regression adjusting for multivariate covariates. We used likelihood ratio tests to calculate $P$ values comparing a model with haplotypes versus a model without. All $P$ values presented are two tailed and were considered to be statistically significant if they were below 0.05 .

\section{Results}

\section{Patient population}

Descriptive data on breast cancer patients included in the study are summarized in Table 2, in which the two case groups are described (patients with localized disease $[n=146]$ and cases patients regional and metastatic disease $[n=154]$ ). The data in Table 2 show that the group with localized disease was about 4 years older than those with advanced disease at diagnosis (mean age at diagnosis: 59.7 years versus 55.2 years; $P=0.0042$ ). Those with localized disease had a smaller 
Table 2

\begin{tabular}{|c|c|c|c|c|}
\hline Characteristic & & Localized $(n=148)$ & Regional/metastatic $(n=152)$ & $P$ \\
\hline \multirow[t]{4}{*}{ Age at diagnosis } & $<50$ years & $44(30.1 \%)$ & $57(37.0 \%)$ & $0.0042^{\mathrm{a}}$ \\
\hline & $\geq 50$ years & $102(69.9 \%)$ & $97(63.0 \%)$ & \\
\hline & Mean age (years; mean \pm SD) & $59.7 \pm 14.3$ & $55.2 \pm 12.6$ & \\
\hline & Range (years) & 33-91 & $25-87$ & \\
\hline \multirow[t]{3}{*}{ ER status } & Positive & $109(74.7 \%)$ & $105(68.2 \%)$ & $0.1741^{b}$ \\
\hline & Negative & $22(15.0 \%)$ & $33(21.4 \%)$ & \\
\hline & Unknown & $15(10.3 \%)$ & $16(10.4 \%)$ & \\
\hline \multirow[t]{3}{*}{ PR status } & Positive & $96(65.7 \%)$ & $87(56.5 \%)$ & $0.0489^{b}$ \\
\hline & Negative & $33(22.7 \%)$ & 451 (33.1\%) & \\
\hline & Unknown & $17(11.6 \%)$ & $16(10.4 \%)$ & \\
\hline \multirow[t]{5}{*}{ Tumor size } & $<2 \mathrm{~cm}$ & $102(69.9 \%)$ & $55(35.7 \%)$ & $<0.0001^{c}$ \\
\hline & $\geq 2 \mathrm{~cm}$ & $34(27.4 \%)$ & $84(54.6 \%)$ & \\
\hline & Unknown & $4(2.7 \%)$ & $15(9.7 \%)$ & \\
\hline & Mean size $(\mathrm{cm} ;$ mean $\pm \mathrm{SD})$ & $1.6 \pm 1.0$ & $3.1 \pm 2.7$ & \\
\hline & Range $(\mathrm{cm})$ & $0.1-6.5$ & $0.4-18.0$ & \\
\hline \multirow[t]{3}{*}{ Grade } & Well to moderate & $86(58.9 \%)$ & $46(29.9 \%)$ & $<0.0001^{b}$ \\
\hline & Poor & $26(17.8 \%)$ & $79(51.3 \%)$ & \\
\hline & Unknown & $34(23.3 \%)$ & $29(18.8 \%)$ & \\
\hline
\end{tabular}

Values are expressed as $n(\%)$, unless otherwise stated. aBased on a t-test. bBased on a Fisher's exact test excluding the missing values. ${ }^{\text {bBased }}$ on a t-test on the logarithmic scale. ER, estrogen receptor; PR, progesterone receptor; SD, standard deviation.

mean tumor size $(1.6 \mathrm{~cm}$ versus $3.1 \mathrm{~cm} ; P<0.0001)$. In addition, the group with localized disease had a higher frequency of well differentiated tumors $(P<0.0001)$. No difference in the distribution of estrogen receptor (ER)-positive tumors and a marginal statistical significant difference in progesterone receptor (PR)-positive tumor status were observed between the two study groups. No statistically significant associations were observed between stage of the disease and other epidemiologic risk factors such as alcohol use, smoking, age at menarche, menopause status, parity, estrogen use, and family history of breast, ovarian, or prostate cancer among firstdegree relatives.

\section{Analysis of SIPA1 single nucleotide polymorphism genotype frequencies}

The allele frequencies for the three SNPs in all breast cancer patients included in the study were 0.359 for SNP -313G $>A$, 0.556 for SNP 545C>T, and 0.144 for SNP 2760G $>$ A. For none of the SNPs did the distribution deviate from HardyWeinberg equilibrium. All three SNPs were in pairwise linkage disequlibrium, as indicated by the parameter D', with $D^{\prime}$ values of 0.94 between rs931127 and rs3741378, 0.85 between rs931127 and rs746429, and 0.86 between rs3741378 and rs746429.
In a univariate analysis between the distribution of the genotypes and tumor characteristics, we observed the following. First, -313G>A SNP genotypes were significantly associated with the presence of positive lymph nodes at diagnosis; 33.3\% of those with GG genotype had positive lymph nodes as compared with $52.7 \%$ among those with GA or AA genotype $(P=0.0139)$. Second, 545C $>$ T was associated with ERnegative tumors, with $15.3 \%$ of those with CC genotype being ER negative as compared with $34.8 \%$ among those with CT or TT genotype $(P=0.0006)$; it was also associated with PRnegative tumors, with $27.8 \%$ of those with CC genotype being PR negative as compared with $42.0 \%$ among those with CT or TT genotype $(P=0.0350)$. This indicated that the presence of the $\mathrm{T}$ allele is associated with a number of indicators of a more aggressive disease process. Third, the frequency distribution of genotypes for the $2760 \mathrm{G}>\mathrm{A}$ SNP revealed a significant association between the presence of the $A A$ or $G A$ genotype and advanced disease and the presence of positive lymph nodes at diagnosis. The combined frequency of homozygote variant $A A$ and heterozygote $G A$ genotypes was significantly higher in those individuals with nonlocalized, advanced disease than in individuals in whom the tumor remained confined to the breast $(65.3 \%$ versus $50.4 \% ; P=$ $0.0154)$. A similar pattern was observed with respect to lymph 
Table 3

\begin{tabular}{|c|c|c|c|c|c|}
\hline \multirow[t]{2}{*}{ SNP genotype } & \multirow{2}{*}{$\begin{array}{l}\text { Disease characteristic } \\
\quad(n[\%])\end{array}$} & \multicolumn{2}{|c|}{ Odds ratios } & \multirow[t]{2}{*}{$95 \% \mathrm{Cl}$} & \multirow[t]{2}{*}{$P$} \\
\hline & & Crude & Adjusteda $^{a}$ & & \\
\hline \multicolumn{6}{|c|}{$-313 G>A$ (rs931127; negative/positive nodes) } \\
\hline GG & $36(27.5) / 18(14.5)$ & 1.00 & 1.00 & & \\
\hline GA & $58(44.3) / 60(48.4)$ & 2.23 & 2.15 & $1.14-4.06$ & 0.0083 \\
\hline AA & $34(28.2) / 46(37.1)$ & & & & \\
\hline \multicolumn{6}{|c|}{ 545C>T (rs3741378; ER positive/ER negative) } \\
\hline $\mathrm{CC}$ & $166(78.7) / 30(55.6)$ & 1.00 & 1.00 & & \\
\hline CT & $39(18.5) / 22(40.7)$ & 2.95 & 2.90 & $1.52-5.48$ & 0.0012 \\
\hline $\mathrm{TT}$ & $6(2.8) / 2(2.0)$ & & & & \\
\hline \multicolumn{6}{|c|}{ 545C >T (rs3741378; PR positive/PR negative) } \\
\hline $\mathrm{CC}$ & $140(77.8) / 54(65.1)$ & 1.00 & 1.00 & & \\
\hline CT & $35(19.4) / 26(31.3)$ & 1.88 & 1.86 & $1.05-3.30$ & 0.0339 \\
\hline $\mathrm{TT}$ & $5(2.8) / 3(3.6)$ & & & & \\
\hline \multicolumn{6}{|c|}{ 2760G>A (rs746429; negative/positive nodes) } \\
\hline GG & $64(49.6) / 41(32.5)$ & 1.00 & 1.00 & & \\
\hline GA & $46(35.7) / 65(51.6)$ & 2.04 & 2.05 & $1.23-3.44$ & 0.0062 \\
\hline AA & $19(14.7) / 20(20.0)$ & & & & \\
\hline
\end{tabular}

aAdjusted analysis models include age at diagnosis. $\mathrm{Cl}$ confidence interval; $\mathrm{ER}$, estrogen receptor; progesterone receptor.

node involvement, in which the combined frequency of homozygote variants (AA) and heterozygotes (GA) was higher in those individuals with metastasis in one or more axillary lymph nodes than in those with no nodal involvement $(64.5 \%$ versus $50.4 \% ; P=0.0074)$. These findings indicate that the variant $A$ allele is associated with a more aggressive disease process.

The genotype frequencies for each of the three SNPs in the study population by tumor characteristics are presented in Table 3. In addition, Table 3 shows the univariate and multivariate-adjusted OR and 95\% confidence interval $(\mathrm{Cl})$ of the tumor characteristics according to the genotypes of the three SNPs, assuming a dominant model. In the multivariable analysis we included age at diagnosis in all models. Thus, for the 313G $>$ A SNP the multivariate OR of the presence of positive lymph nodes was 2.15 (95\% Cl 1.14-4.06; $P=0.0083)$. This implies that an individual with the GA or AA genotype was 2.15 times more likely to have positive lymph nodes than was an individual with GG genotype. The multivariable OR of the $545 \mathrm{C}>\mathrm{T}$ allele and ER and PR status were $2.90(95 \% \mathrm{Cl}$ $1.52-5.48 ; P=0.0012)$ and $1.86(95 \% \mathrm{Cl} 1.05-3.30 ; P=$ $0.0339)$, respectively. Similarly, for the $-2760 G>A$ SNP the multivariate $\mathrm{OR}$ of the presence of positive lymph nodes was $2.05(95 \% \mathrm{Cl} 1.23-3.44 ; P=0.0062)$. This implies that an individual with the GA or AA genotype was 2.05 times more likely to have positive lymph nodes than was an individual with the GG genotype.

\section{Analysis of SIPA1 single nucleotide polymorphism haplotype frequencies}

We investigated the association between haplotypes defined by these three SNPs and the presence of positive lymph nodes, and ER and PR status. There were a total of seven haplotypes that were imputed from the data on 260 patients with complete genotypes on all three SNPs. However, four of those haplotypes accounted for more than $98 \%$ of the observations. Table 4 summarizes the estimated frequencies of the predicted haplotypes, and Table 5 presents the haplotype-specific ORs of the association of haplotypes and the presence of positive lymph nodes, and ER and PR status compared with the most common haplotype (haplotype 1). Haplotypes with allele frequency below $1 \%$ were included with the baseline in our analysis. In the analysis of the presence of positive lymph nodes and haplotypes, the haplotype specific OR for haplotype 3 was $2.20(95 \% \mathrm{Cl} 1.27-3.81 ; P=0.0051)$ compared with haplotype 1 . Thus, women with the estimated haplotype 3 were 2.20 times more likely to present with positive lymph nodes than were women with haplotype 1. In the analysis of ER status and haplotypes, the haplotype specific OR for hap- 
lotype 4 was $2.85(95 \% \mathrm{Cl} 1.36-5.94 ; P=0.0053)$ compared with haplotype 1 . Thus, women with the estimated haplotype 4 were 2.85 times more likely to present with an ERnegative tumor than were women with haplotype 1 . In the analysis of PR status and haplotypes, the haplotype-specific OR for haplotype 4 was $2.16(95 \% \mathrm{Cl} 1.13-4.12 ; P=0.0199)$ compared with haplotype 1 . Thus, women with the estimated haplotype 4 were 2.16 times more likely to present with a PRnegative tumor than were women with haplotype 1 .

\section{Discussion}

In this preliminary study we observed strong associations between the three SIPA1 SNPs and indicators of aggressive breast cancer in this population. Specifically, we report a number of particularly interesting findings regarding nodal status at the time of diagnosis and tumor sex hormone receptor status; these findings suggest that SIPA1 germline variation contributes to metastatic potential in breast cancer. With regard to lymph node involvement, the variant $-313 \mathrm{G}>\mathrm{A}$ and $2760 \mathrm{G}>\mathrm{A}$ alleles were strongly associated with axillary node involvement at the time of diagnosis on univariate analysis. Indeed, the apparent effect of these SNPs on the frequency of metastasis that was evident upon univariate analysis was complemented by the outcome of multivariate and haplotype frequency estimation. Multivariate analysis of genotype frequencies showed that possession of either of the $-313 G>A$ or $2760 G>A$ variant alleles was associated with a significantly increased likelihood of nodal involvement. Similarly, haplotype analyses demonstrated that the haplotype consisting of the wild-type allele of $545 \mathrm{C}>\mathrm{T}$ and the variant alleles of the two other studied SNPs (haplotype 3; Table 5) is associated with nodal involvement at the time of diagnosis. This apparent association with nodal involvement at the time of diagnosis is particularly interesting given that there was no evidence of association of SIPA1 genotype status and tumor size. It was previously demonstrated that a linear relation exists between tumor diameter and the percentage of cases with positive lymph node involvement [18]. One would therefore expect similar associations with tumor size, given the apparent rela- tionship with nodal status at diagnosis. However, this was not the case in the present study, which may well be a consequence of inadequate statistical power to detect such an association. Alternatively, SIPA1 polymorphism might facilitate nodal metastasis independent of tumor size. Whichever is the case, further analysis of the significance of these polymorphisms in different populations is required to clarify the situation.

SIPA1 polymorphisms in this population were also associated with other features of tumors with aggressive behavior. Positive sex hormone receptor status correlates with favorable prognostic features, including a lower rate of cell proliferation and histological evidence of tumor differentiation [19]. The $545 \mathrm{C}>\mathrm{T}$ variant allele was associated with ER-negative and PR-negative tumors in this cohort. In addition, haplotype 4, which is defined by the variant allele $545 \mathrm{C}>\mathrm{T}$, is strongly associated with ER and PR tumor status (Table 5). This again highlights the deleterious effect that SIPA1 germline variation has on prognosis in primary breast carcinoma and implies that variant forms of this gene are associated with more aggressive disease forms.

The importance of these data is heightened when one considers the outcome of our linkage and functional studies of Sipa1 in a murine population $[12,13,20]$, coupled with consideration of the known physiologic functions of SIPA1. Traditional dogma has stated that cancerous cells gain the ability to metastasize as a consequence of somatic mutation (for example, through loss of heterozygosity or epigenetic events). However, it has become increasingly accepted that hereditary factors influence all stages of tumor pathogenesis in apparently sporadic cancers. These heritable defects will be of low penetrance, but hypothetically they could affect any part of the neoplastic cascade, including modulation of metastatic efficiency. Evaluation of quantitative trait loci in inbred mouse models has proven a particularly powerful means of studying the effects of this type of genetic variation. The present study is an additional example of how mouse quantitative trait locus

Table 4

Estimated frequencies of predicted haplotypes

\begin{tabular}{lcccc}
\hline Haplotype & $-313 \mathrm{G}>\mathrm{A}(\mathrm{rs} 931127)$ & 545C>T (rs3741378) & 2760G $>$ A (rs746429) & Estimated frequency (\%) \\
\hline 1 & $\mathrm{G}$ & $\mathrm{C}$ & $\mathrm{G}$ & 42.1 \\
2 & $\mathrm{~A}$ & $\mathrm{C}$ & $\mathrm{G}$ & 8.0 \\
3 & $\mathrm{~A}$ & $\mathrm{C}$ & $\mathrm{A}$ & 34.6 \\
4 & $\mathrm{~A}$ & $\mathrm{~T}$ & $\mathrm{G}$ & $\mathrm{A}$ \\
$5^{\mathrm{a}}$ & $\mathrm{G}$ & $\mathrm{C}$ & $\mathrm{G}$ & 0.9 \\
$5^{\mathrm{a}}$ & $\mathrm{G}$ & $\mathrm{T}$ & $\mathrm{A}$ & 0.5 \\
$5^{\mathrm{a}}$ & $\mathrm{A}$ & $\mathrm{T}$ & $\mathrm{A}$ & 0.3 \\
\hline
\end{tabular}

Haplotypes frequencies were estimated from the 260 samples with complete genotype data on all three polymorphisms. ${ }^{\text {al }}$ aplotypes with an estimated frequency below $1 \%$ were pooled for the purposes of analysis. 
Table 5

\begin{tabular}{|c|c|c|c|c|c|c|}
\hline \multirow[t]{2}{*}{ Haplotype $^{\mathrm{a}}$} & \multicolumn{2}{|c|}{ Positive nodes } & \multicolumn{2}{|c|}{ ER status } & \multicolumn{2}{|c|}{ PR status } \\
\hline & OR $(95 \% \mathrm{Cl})$ & $P$ & OR $(95 \% \mathrm{Cl})$ & $P$ & OR $(95 \% \mathrm{Cl})$ & $P$ \\
\hline 1 & 1.00 & & 1.00 & & 1.00 & \\
\hline 2 & $1.14(0.52-2.51)$ & 0.7410 & $0.93(0.34-2.60)$ & 0.8961 & $0.99(0.42-2.33)$ & 0.9828 \\
\hline 3 & $2.20(1.27-3.81)$ & $0.0051^{*}$ & $0.62(0.30-1.29)$ & 0.2005 & $1.50(0.82-2.77)$ & 0.1915 \\
\hline 4 & $1.00(0.54-1.86)$ & 0.9940 & $2.85(1.36-5.94)$ & $0.0053^{\star}$ & $2.16(1.13-4.12$ & $0.0199^{*}$ \\
\hline
\end{tabular}

aHaplotypes with an estimated frequency under $1 \%$ were pooled within the baseline for the purposes of analysis. * Statistically significant comparisons of haplotype frequencies.

data, when used in an appropriate manner, can help to unravel the complexities of hereditary influences in sporadic human cancer [21].

Although the specific mechanism by which SIPA1 modulates metastatic efficiency is currently unclear, it is worthwhile to speculate on how observations of SIPA1 functionality in mice and humans may relate to the process of tumor progression and characterization. Specifically, tumor development in a background of germline-encoded SIPA1 dysfunction may enhance the ability of tumor cells to escape the primary lesion through alteration in cell morphology/polarity and/or by weakening the strength of intercellular contacts. This argument gains further potency when one considers the negative regulatory effect of SIPA1 on Rap1, and that Rap1 has been implicated in the maintenance of epithelial polarization [22] as well as in the maintenance of intercellular adherens junctions [23]. Further studies will be required to confirm whether this is the case.

SIPA1 has been shown to have GTPase activity that is specific for Rap1 and Rap2, both of which are members of the Ras family of GTPases, and are involved in regulating cell proliferation, differentiation, and adhesion [24]. Indeed, SIPA1 appears to play a prominent role in regulating cell adhesion, and transient expression of this gene in HeLa cells induces cellular rounding up and detachment from the culture surface [25]. It appears to act by modulating a number of cellular adhesion molecules, for example $\beta_{1}$-integrin, and thus regulates the interactions between these molecules and other cellular and extracellular matrix proteins involved in the process of adhesion [26-28]. It has been hypothesized that SIPA1 negatively regulates integrin-mediated cell adhesion via Rap1 GAP activity [27]. Specifically, SIPA1 interacts with the cytoskeletalanchoring protein AF6 through a common protein-protein interaction motif known as a 'PDZ domain' [27].

In addition to the effects on intercellular adhesion, disruption of SIPA1 has been shown to have potent effects on cellular proliferation. For example, SIPA1-deficient mice develop a spectrum of symptoms resembling those seen in human myelodysplastic and myeloproliferative disorders $[29,30]$. This is especially noteworthy because metastatic cells must be able to proliferate effectively at a distant site in what must be considered a foreign microenvironment, if they are to become a clinically relevant metastatic lesion. When all this evidence is considered, we argue that functional data from both mouse and human studies strongly support the findings of the present case-only study, in which we demonstrate that germline polymorphisms and haplotypes of SIPA1 are associated with enhanced metastatic efficiency in primary breast carcinoma.

However, further experiments will be required to confirm the role of SIPA1 germline variation in modulating tumor progression efficiency, the most obvious of which is to replicate the associations seen here in other populations. It is also vital to resequence various elements of the SIPA1 gene to attempt to identify novel polymorphisms, the most critical of which is that encoding the PDZ protein-protein interaction domain - a region that is critical to SIPA1 functionality [27]. This endeavor is especially important given that the exon $122760 \mathrm{G}>\mathrm{A}$ SNP - a noncoding, synonymous polymorphism - is strongly associated with aggressive disease in this study. This may well be a reflection of the physical proximity of this SNP to the other polymorphisms characterized in the study, which could have a more plausible effect on SIPA1 functionality. This hypothesis is somewhat supported when one considers that a haplotype containing the $2760 \mathrm{G}>\mathrm{A}$ variant allele is associated with more aggressive disease at diagnosis, as measured by positive lymph node involvement. Conversely, it may well be that the $2760 G>A$ SNP is in linkage disequilibrium with a nearby yet uncharacterized SNP with a more obvious functional effect. Indeed, one could postulate that the associations observed in the present study are in fact a consequence of another gene in linkage disequilibrium with SIPA1, because the functional significance (if any) of each of the polymorphisms has not been assessed. However, we argue that this is unlikely because we investigated genes in the chromosomal regions surrounding Sipa 1 in the mouse and found no data supporting the hypothesis that surrounding genes are involved in modu- 
lating metastatic efficiency [13]. This, in combination with observations relating to Sipa 1 functionality in the mouse and the fact that human haplotype blocks are probably significantly smaller than in inbred mice, leads to the conclusion that the observed associations are a result of SIPA1 polymorphism.

The implications of this work are potentially far reaching in that it may well prove possible to assess metastatic potential through genotyping polymorphisms in germline DNA. The ability to evaluate the risk for metastatic disease from readily available tissues such as blood could be a powerful means to augment currently available means of assessing prognosis in breast cancer. However, we do acknowledge that the effect of germline SIPA1 variation in isolation is probably too low to allow for the development of an assay that would possess sufficient sensitivity and specificity to determine metastatic propensity accurately. However, it is plausible that a panel of polymorphisms derived from a number of metastasis 'susceptibility' genes would possess the power and accuracy to enable the development of a clinically relevant prognostic assay, which will necessitate the identification of further metastasis efficiency modifier genes.

\section{Conclusion}

Our results imply that SIPA1 germline polymorphisms are associated with aggressive disease behavior in the present cohort. If the results of this study hold true in other populations, then the potential for this to have a positive impact on current breast cancer staging and treatment protocols may be great. For example, staging of primary breast cancer in cases in which there is no palpable evidence of nodal metastasis typically requires axillary dissection to assess nodal status. However, controversy exists as to the actual benefit of nodal sampling in those patients with small tumors because of the morbidity frequently associated with this procedure. For example, the incidence of axillary node relapse in patients with stage T1a tumors (tumor $>0.1 \mathrm{~cm}$ but not $>0.5 \mathrm{~cm}$ in greatest dimension) treated without axillary dissection was $2 \%$ [31]. It is plausible that analysis of SIPA1 polymorphisms could be used as an additional element of the staging process that might allow axillary node-negative patients with small primary tumors (for example, T1-2 N0) to be classed as being at increased risk for development of metastasis. In turn, this could then become a powerful influence on whether to initiate adjuvant therapy in patients who would not currently be considered for this type of treatment.

\section{Competing interests}

The authors declare that they have no competing interests.

\section{Authors' contributions}

NC was responsible for genotyping polymorphisms, contributed to study design, and drafted the manuscript. The University of California Irvine investigators played several roles in this report. HA-C contributed to the study design, the recruitment of subjects and the interviewing and biospecimen collection, and drafting and editing of the manuscript. AZ contributed to the study design, recruitment of subjects and data analysis, and drafting and editing of the manuscript. DP contributed to study design, biospecimen processing, and drafting and editing of the manuscript. JH participated in data processing and analysis, and editing of the manuscript. $\mathrm{KH}$ participated in study design, was responsible for supervising laboratory work, and was involved in manuscript preparation. All authors read and approved the final manuscript.

\section{Acknowledgements}

We should like to thank Fabienne Lesueur for kindly providing the sequences of the fluorogenic minor groove binder TaqMan probes used in this study. We should also like to thank Dr Chad Garner for his help in the analysis.

This research was supported (in part) by the Intramural Research Program of the $\mathrm{NIH}$, National Cancer Institute, Center for Cancer Research. We would also like to acknowledge support for this research by funding from the $\mathrm{NCl}$ (CA58860 and CA 58860-09), the University of California Breast Cancer Research Program (404120), and the Lon Smith Foundation (LVS-35977).

\section{References}

1. Jemal A, Tiwari RC, Murray T, Ghafoor A, Samuels A, Ward E, Feuer EJ, Thun MJ: Cancer statistics, 2004. CA Cancer J Clin 2004, 54:8-29.

2. Guarneri V, Conte PF: The curability of breast cancer and the treatment of advanced disease. Eur J Nucl Med Mol Imaging 2004, Suppl 1:S149-161.

3. Chung CT, Carlson RW: Goals and objectives in the management of metastatic breast cancer. Oncologist 2003, 8:514-520.

4. Houlston RS, Peto J: The search for low-penetrance cancer susceptibility alleles. Oncogene 2004, 23:6471-6476.

5. Foulkes WD, Rosenblatt J, Chappuis PO: The contribution of inherited factors to the clinicopathological features and behavior of breast cancer. J Mammary Gland Biol Neoplasia 2001, 6:453-465.

6. Goode EL, Dunning AM, Kuschel B, Healey CS, Day NE, Ponder $B A$, Easton DF, Pharoah PP: Effect of germ-line genetic variation on breast cancer survival in a population-based study. Cancer Res 2002, 62:3052-3057.

7. Robson ME, Chappuis PO, Satagopan J, Wong N, Boyd J, Goffin JR, Hudis C, Roberge D, Norton L, Begin LR, et al:: A combined analysis of outcome following breast cancer: differences in survival based on BRCA1/BRCA2 mutation status and administration of adjuvant treatment. Breast Cancer Res 2004, 6:R8-R17.

8. Foulkes WD, Wong N, Brunet JS, Begin LR, Zhang JC, Martinez JJ, Rozen F, Tonin PN, Narod SA, Karp SE, et al:: Germ-line BRCA1 mutation is an adverse prognostic factor in Ashkenazi Jewish women with breast cancer. Clin Cancer Res 1997, 3:2465-2469.

9. Stoppa-Lyonnet D, Ansquer Y, Dreyfus H, Gautier C, Gauthier-Villars M, Bourstyn E, Clough KB, Magdelenat $H$, Pouillart P, VincentSalomon $A$, et al:: Familial invasive breast cancers: worse outcome related to BRCA1 mutations. J Clin Oncol 2000, 18:4053-4059.

10. Lifsted T, Le Voyer T, Williams M, Muller W, Klein-Szanto A, Buetow $\mathrm{KH}$, Hunter $\mathrm{KW}$ : Identification of inbred mouse strains harboring genetic modifiers of mammary tumor age of onset and metastatic progression. Int J Cancer 1998, 77:640-644.

11. Guy CT, Cardiff RD, Muller WJ: Induction of mammary tumors by expression of polyomavirus middle $\mathrm{T}$ oncogene: a transgenic mouse model for metastatic disease. Mol Cell Biol 1992, 12:954-961.

12. Hunter KW, Broman KW, Voyer TL, Lukes L, Cozma D, Debies MT, Rouse J, Welch DR: Predisposition to efficient mammary tumor 
metastatic progression is linked to the breast cancer metastasis suppressor gene Brms1. Cancer Res 2001, 61:8866-8872.

13. Park YG, Zhao X, Lesueur F, Lowy DR, Lancaster M, Pharoah P, Qian X, Hunter KW: Sipa1 is a candidate for underlying the metastasis efficiency modifier locus Mtes1. Nat Genet 2005, 37:1055-1062

14. Ziogas A, Gildea M, Cohen P, Bringman D, Taylor TH, Seminara D, Barker D, Casey G, Haile R, Liao SY, et al.: Cancer risk estimates for family members of a population-based family registry for breast and ovarian cancer. Cancer Epidemiol Biomarkers Prev 2000, 9:103-111.

15. Single nucleotide polymorphisms [http://www.ncbi.nlm.nih.gov/ SNP/]

16. Crawford NP, Colliver DW, Funke AA, Young MN, Kelley S, Cobbs GA, Petras RE, Galandiuk S: Characterization of genotype-phenotype relationships and stratification by the CARD15 variant genotype for inflammatory bowel disease susceptibility loci using multiple short tandem repeat genetic markers. Hum Mutat 2005, 25:156-166.

17. Zaykin DV, Westfall PH, Young SS, Karnoub MA, Wagner MJ, Ehm MG: Testing association of statistically inferred haplotypes with discrete and continuous traits in samples of unrelated individuals. Hum Hered 2002, 53:79-91.

18. Carter CL, Allen C, Henson DE: Relation of tumor size, lymph node status, and survival in 24,740 breast cancer cases. Cancer 1989, 63:181-187.

19. Platet N, Cathiard AM, Gleizes M, Garcia M: Estrogens and their receptors in breast cancer progression: a dual role in cancer proliferation and invasion. Crit Rev Oncol Hematol 2004, 51:55-67.

20. Park YG, Clifford R, Buetow KH, Hunter KW: Multiple cross and inbred strain haplotype mapping of complex-trait candidate genes. Genome Res 2003, 13:118-121.

21. Ewart-Toland A, Briassouli $P$, de Koning JP, Mao JH, Yuan J, Chan F, MacCarthy-Morrogh L, Ponder BA, Nagase $\mathrm{H}$, Burn J, et al.: Identification of Stk6/STK15 as a candidate low-penetrance tumor-susceptibility gene in mouse and human. Nat Genet 2003, 34:403-412.

22. Ohba Y, Ikuta K, Ogura A, Matsuda J, Mochizuki N, Nagashima K, Kurokawa K, Mayer BJ, Maki K, Miyazaki J, et al.: Requirement for C3G-dependent Rap1 activation for cell adhesion and embryogenesis. EMBO J 2001, 20:3333-3341.

23. Yajnik V, Paulding C, Sordella R, McClatchey Al, Saito M, Wahrer DC, Reynolds P, Bell DW, Lake R, van den HS, et al.: DOCK4, a GTPase activator, is disrupted during tumorigenesis. Cell 2003, 112:673-684.

24. Kurachi $H$, Wada $Y$, Tsukamoto $N$, Maeda M, Kubota $H$, Hattori $M$, Iwai $\mathrm{K}$, Minato $\mathrm{N}$ : Human SPA-1 gene product selectively expressed in lymphoid tissues is a specific GTPase-activating protein for Rap1 and Rap2. Segregate expression profiles from a rap1GAP gene product. J Biol Chem 1997, 272:28081-28088.

25. Tsukamoto N, Hattori M, Yang H, Bos JL, Minato N: Rap1 GTPase-activating protein SPA-1 negatively regulates cell adhesion. J Biol Chem 1999, 274:18463-18469.

26. Liu L, Schwartz BR, Tupper J, Lin N, Winn RK, Harlan JM: The GTPase Rap1 regulates phorbol 12-myristate 13-acetatestimulated but not ligand-induced beta 1 integrin-dependent leukocyte adhesion. J Biol Chem 2002, 277:40893-40900.

27. Su L, Hattori M, Moriyama M, Murata N, Harazaki M, Kaibuchi K, Minato N: AF-6 controls integrin-mediated cell adhesion by regulating Rap1 activation through the specific recruitment of Rap1GTP and SPA-1. J Biol Chem 2003, 278:15232-15238.

28. Shimonaka M, Katagiri K, Nakayama T, Fujita N, Tsuruo T, Yoshie $\mathrm{O}$, Kinashi T: Rap1 translates chemokine signals to integrin activation, cell polarization, and motility across vascular endothelium under flow. J Cell Biol 2003, 161:417-427.

29. Ishida $D$, Kometani $\mathrm{K}$, Yang $\mathrm{H}$, Kakugawa $\mathrm{K}$, Masuda $\mathrm{K}$, Iwai $\mathrm{K}$, Suzuki M, Itohara S, Nakahata T, Hiai H, et al.: Myeloproliferative stem cell disorders by deregulated Rap1 activation in SPA-1deficient mice. Cancer Cell 2003, 4:55-65.

30. Largaespada DA: A bad rap: Rap1 signaling and oncogenesis. Cancer Cell 2003, 4:3-4.

31. Greco M, Agresti R, Cascinelli N, Casalini P, Giovanazzi R, Maucione A, Tomasic G, Ferraris C, Ammatuna M, Pilotti S, et al:: Breast cancer patients treated without axillary surgery: clinical implications and biologic analysis. Ann Surg 2000, 232:1-7. 